\title{
1 A comprehensive evaluation of CRISPR lineage recorders using TraceQC
}

2 Jingyuan $\mathrm{Hu}^{1,2}$, Hyun-Hwan Jeong ${ }^{3}$, Rami Al-Ouran ${ }^{2,4}$, Igor Bado ${ }^{5,6,7}$, Weijie Zhang ${ }^{5,6,7}$, Xiang

3 Zhang ${ }^{5,6,7}$, Zhandong $\mathrm{Liu}^{2,4, *}$

4

$5{ }^{1}$ Program in Quantitative and Computational Biosciences, Baylor College of Medicine,

$6 \quad$ Houston, TX 77030, USA

$7 \quad{ }^{2}$ Jan and Dan Duncan Neurological Research Institute, Texas Children's Hospital, Houston, TX

877030, USA

$9 \quad{ }^{3}$ Center for Precision Health, School of Biomedical Informatics, the University of Texas Health

10 Science Center at Houston, Houston, TX 77030, USA

$11{ }^{4}$ Department of Pediatrics, Baylor College of Medicine, Houston, TX 77030, USA

$12{ }^{5}$ Lester and Sue Smith Breast Center, Baylor College of Medicine, Houston, TX 77030, USA

$13{ }^{6}$ Dan L. Duncan Cancer Center, Baylor College of Medicine, Houston, TX 77030, USA

$14{ }^{7}$ Department of Molecular and Cellular Biology, Baylor College of Medicine, Houston, TX

1577030, USA

16

$17{ }^{*}$ Corresponding author.

18 E-mail: zhandonl@bcm.edu

\section{Abstract}

The CRISPR-Cas9 genome editing-based lineage tracing system is emerging as a

22 powerful tool to track cell lineages at unprecedented scale and resolution. However, the

23 complexity of CRISPR-Cas9 induced mutations has raised challenges in lineage reconstruction, 
24 which requires a unique computational analysis framework. Meanwhile, multiple distinctive

25 CRISPR-based high-throughput lineage recorders have been developed over the years in which

26 the data analysis is incompatible across platforms. To address these challenges, first, we present

27 the TraceQC, a cross-platform open-source package for data processing and quality evaluation of

28 CRISPR lineage tracing data. Second, by using the TraceQC package, we performed a

29 comprehensive analysis across multiple CRISPR lineage recorders to uncover the speed and

30 distribution of CRISPR-induced mutations. Together, this work provides a computational

31 framework for the CRISPR lineage tracing system that should broadly benefit the design and

32 application of this promising technology.

\section{Introduction}

Determining the origin of cells in multicellular organisms is a long-term goal in

36 developmental biology. Recent advancements in CRISPR-Cas9 genome editing have brought a

37 new generation of lineage tracing techniques that can simultaneously mark cells with irreversible

38 mutations $^{1-3}$. In general, these techniques use CRISPR-Cas9 genome editing to target a specific

39 DNA barcode, which generates diversified mutations that can simultaneously track the cell

40 developmental process. When combined with the state-of-art single-cell sequencing, this lineage

41 tracing technique enables tracking tens of thousands of cells in one experiment. This massive

42 scale lineage tracing has opened up new opportunities to study cell development in single-cell

43 resolution. For example, several studies have applied this technique to map the clonal evolution

44 of cancer metastasis ${ }^{4-7}$.

46 undergoes rapid expansion. Up to now, several lineage tracing platforms have been developed. 
47 GESTALT is one of the first platforms that implemented the idea of CRISPR lineage recording

48 by engineering a target array into the zebrafish's genome ${ }^{8}$. Using the CRISPR introduced

49 mutations, the GESTALT mapped a complete tissue-level developmental tree of zebrafish.

50 Subsequently, several studies have integrated the CRISPR lineage recorder with single-cell RNA

51 sequencing to provide a cell-level lineage mapping of zebrafish ${ }^{9-11}$ and mouse ${ }^{12,13}$. While most

52 recorders used the canonical CRISPR system, the homing guide RNA (hgRNA) lineage recorder

53 made a major modification by directing the Cas9-hgRNA complex to cuts the DNA locus of

54 itself $^{14}$. This modification has increased the efficiency and diversity of CRISPR genome

55 editing ${ }^{15,16}$.

Across all the CRISPR lineage recorders, the design of the DNA barcodes is vastly

57 different. Some distinctive categories are: 1) Synthesized target array ${ }^{8,9,12,13}$. 2) The hgRNA

58 system $\left.^{15,16} .3\right)$ Specific DNA sequences that pre-exist in the genome ${ }^{10,11,17}$ (Fig. 1A). Although

59 each platform has different design philosophy on DNA barcode, one commonality is that the

60 barcode sequences are usually compact (less than 300-bp), which makes the high-throughput

61 sequencing readout very homogeneous. As a result, the sequencing analysis could be compatible

62 across multiple CRISPR-based lineage tracing technologies.

63 In general, it takes two major steps to reconstruct the lineage tree. Step one is to identify

64 the CRISPR-induced mutations from the sequencing data. A major characteristic of CRISPR-

65 induced mutations is the prevalence of insertions and deletions (indels) ${ }^{18}$. This is primarily a

66 consequence of non-homologous end joining (NHEJ) mechanisms when it repairs the Cas9-

67 induced double-strand break (DSB) ${ }^{19,20}$. To extract indels (ranging from 1-bp to 20-bp) that

68 prevalently exists in the sequencing data, sequencing alignment with affine gap penalty is

69 commonly used. Another characteristic of CRISPR-induced mutations is that the mutation 
70 patterns are unevenly distributed. When the Cas9-guide RNA complex cuts the target sequence,

71 the cleavage location is usually a few base pairs upstream to the PAM sequence ${ }^{21-22}$. As a result,

72 the NHEJ repaired DNA sequence is highly deterministic ${ }^{23}$. In fact, the most frequent mutation

73 outcome of the canonical guide RNA system could contribute $10 \%-80 \%$ of the entire library ${ }^{24}$,

74 which causes a massive scale of parallel evolution (cells from independent lineage acquire the

75 same mutation) of CRISPR barcodes. Therefore, a crucial part of sequencing analysis is to

76 understand the underlying mutation rate caused by NHEJ, which could alleviate the false

77 positivity caused by parallel evolution.

78 When mutations are extracted from the DNA barcode sequence, step two is

79 reconstructing the lineage tree using mutations. Although classical phylogenetic algorithms such

80 as maximum parsimony ${ }^{25,26}$ are used to reconstruct the large-scale lineage tree ${ }^{8}$, they are not

81 designed to capture the key properties of CRISPR-induced mutations. For example, unlike

82 somatic mutations, CRISPR-induced deletions are typically irreversible. Also, the root of the

83 lineage tree is given by the unmutated sequence, which is usually unobtainable in phylogenetic

84 studies. Since classical phylogenetic algorithms do not consider these characteristics, several

85 studies have designed next-generation lineage reconstruction algorithms using statistical

86 modeling ${ }^{27-30}$, including an open competition $^{31}$.

87 Although step two is arguably more computationally challenging, a good lineage

88 reconstruction strategy should integrate the two steps seamlessly. However, sequencing analysis

89 is often-times neglected in the lineage reconstruction methodology. For example, LinTIMaT $^{27}$

90 and GAPML ${ }^{28}$ are two novel reconstruction algorithm that uses maximum likelihood to estimate

91 the lineage tree of GESTALT. Although both methods have considered unique aspects of lineage

92 tree building, such as the importance of mutation rate in parallel evolution, their methodology is 
93 purely based on the GESTALT's mutation metadata. As a result, the connections between the

94 computational model and real experimental data is inexplicit. On the other hand, Cassiopeia ${ }^{29}$, a

95 modified maximum parsimony-based reconstruction methodology, has provided raw sequencing

96 data processing pipeline prior to the reconstruction algorithm. But similarly, the sequencing

97 analysis is secondary in Cassiopeia, in which limited results are shown. We believe a

98 comprehensive sequencing analysis of CRISPR lineage tracing data could benefit these

99 methodologies.

There are several critical challenges in the processing of the CRISPR lineage tracing

101 dataset.1) The CRISPR mutation outcome is highly deterministic, which causes a massive level

102 of parallel evolutions in many lineage recorders. Parallel evolution of barcodes is a significant

103 source of false positives in lineage tracing yet not systematically evaluated in lineage tracing

104 datasets. Research has shown that the CRISPR mutation outcomes could be captured using

105 sequences only, which could be directly used to derive the mutation rate of lineage recorders. 2)

106 The CRISPR editing intensity could significantly influence the lineage recording. For example,

107 intensive CRISPR editing can cause the DNA barcode to saturate too early, preventing the

108 system from recording lineages at a later stage. 3) Since these current methodologies are only

109 performed on one particular dataset, it would be difficult to apply to another CRISPR-based

110 lineage recorder. Moreover, a unified data format for CRISPR tracing data is critically missing,

111 causing the lineage reconstruction strategies incompatible across platforms. Therefore, combing

112 these methodologies with a standardized sequencing analysis pipeline could enable a broader

113 application of the methods across multiple lineage recorders.

Toward this end, we have developed the TraceQC package, which provides a cross-

115 platform data processing pipeline and quality evaluation of multiple sequencing-based CRISPR 
116 lineage tracing platforms. Next, by applying the TraceQC package, we have evaluated the key

117 properties of CRISPR mutations across several lineage recorders. Together, the TraceQC

118 package provides a general framework to understand this unique type of data, thereby promoting

119 the application of CRISPR lineage tracing techniques.

121 Definitions / Glossary

122 Target: the DNA sequence that a Cas9-gRNA complex can edit.

123 DNA barcode / barcode: the DNA sequence used to record lineage. It may be composed of a

124 single target or a target array, depending on the specific lineage tracing platform.

125 Mutation: an edit in the DNA barcode caused by CRISPR-Cas9.

126 Mutation rate: the proportion of a particular mutation in the entire mutation profile. It measures

127 the likelihood of a particular mutation outcome.

128 Mutation speed: the number of CRISPR-induced mutations generated in a given period of time.

129 It measures how quick CRISPR edits the DNA barcode. This is referred as rate decay in the 130 GAPML paper.

\section{Results}

134 TraceQC identifies CRISPR-induced mutations from sequencing data. The TraceQC

135 pipeline takes raw sequencing data as input and extracts mutations from each sequence (Fig. 1B, 136 Methods). Briefly, Needleman-Wunsch alignment is performed on raw sequences. Due to the 137 nature of the NHEJ DNA repair mechanism, indels are prevalent in CRISPR-induced mutations. 138 Here, we decided to set a small gap opening and extension penalty in the alignment algorithm of 
139 TraceQC to favor insertion and deletion over substitution. Next, each aligned sequence is

140 indexed according to the reference barcode in which location information is extracted for each

141 mutation. To reduce technical artifacts (e.g. PCR error, sequencing error, sample contamination),

142 the TraceQC package denoises the dataset using filters based on read count and alignment score

143 (Fig. 1C, Methods). Finally, the package provides a series of functions that evaluate the mutation

144 outcomes across multiple platforms (Fig. 1D, Fig. S13-S28). To illustrate the robustness of the

145 TraceQC package, we analyzed the dataset from all major platforms (Table 1) and revealed

146 differences in mutation characteristics such as speed and rate.

149 most platforms exclude substitutions from the mutation library because they are not as prevalent as indels in CRISPR-induced mutations, it is a viable outcome of NHEJ ${ }^{32-34}$. Using the TraceQC

151 pipeline, we detected the three mutation types: insertion, deletion, and substitution vastly exist in 152 every lineage recorder. On average, three mutations types contribute to a similar diversity to the 153 entire mutation library (Fig. 2A). However, deletion is the most prominent mutation type among 154 the three, as it has the longest length per sequence (Fig. 2B). Each lineage recorder has indel 155 length preference due to differences in experimental designs (Fig. 2C). For example, the barcode 156 sequences of GESTALT and Carlin is a synthesized target array in which deletions can be 157 classified into intra-target deletion and inter-target deletion. While the length of intra-target 158 deletion is mostly less than 10-bp in both GESTALT and Carlin, the length of inter-target 159 deletion could reach 200-bp (Fig S1). In contrast, LINNAEUS is a single-target platform in which CRISPR is used to target red fluoresce protein transgene. Therefore, the targetable region 161 of LINNAEUS has shorter length than target array and we observed that most mutations are 
concentrated upstream to the PAM sequence (Fig. 2D). Although the homing-guide RNA system is also a single-target platform, its average deletion length is longer than LINNAEUS due to

164 deletions spanning into the extended homing-guide scaffold regions downstream to the PAM 165 sequence (Fig. S9). Contrary to deletion, the pattern of insertion is more consistent across platforms. In

167 Carlin, GESTALT, and hgRNA system, the most frequent insertion length is 1-bp. Large insertions that are more than 16-bp are rarely seen (Fig. 2C). The mutation properties discovered

169 by the TraceQC are concordant with the original research, and therefore demonstrate that the

170 TraceQC pipeline has provided accurate cross-platform data analysis.

172 Impact of mutation speed on the accuracy of lineage reconstruction. Genome editing-based

173 lineage tracing technology requires the accumulation of mutations over time. In current CRISPR

174 lineage recorders, the process of mutation generation is presumably independent of cell division.

175 Therefore, a sound lineage recorder should synchronize the cell division rate with the mutation

176 generations rate such that enough signals are generated to track cell development. Ideally, the

177 mutation speed should be kept moderate. While slow mutation speed will not produce enough

178 signals to mark every cell, quick mutation speed will exhaust the CRISPR barcode too quickly to 179 record a prolonged process (Fig. 3A).

181 Adjusting mutation speed in the CRISPR-Cas9 system. Presumably, the mutation speed of

182 CRISPR is determined by the binding efficiency between target sequence and the Cas9 protein.

183 A study has shown that the target sequence, especially the PAM-proximal region is a major

184 determinant of Cas9-target binding efficiency ${ }^{39}$. For example, in the Carlin's target array, each 
target can be edited by a perfectly matched guide RNA, which results in similar mutation speed across multiple targets (Fig. S2). However, modifications on the target sequence or sgRNA sequence could decrease mutation speed ${ }^{39,40}$. In the hgRNA-invitro system, researchers engineered the B-21 barcode with multiple PAM binding sites that decreases the mutation speed $^{41}$ (Fig. S3). Besides, the hgRNA system has further synthesized barcodes with various lengths to adjust the mutation speed (Fig. S3 - S4). The result shows the mutation speed becomes slower when there is an increase on the barcode length upstream to the spacer. Although

192 designing a specific target sequence or intentionally creating an off-target event can adjust the 193 mutation speed, these effects are mostly constitutional. Once the target sequences are determined 194 in the system, it is hard to further synchronize them with cell divisions rate.

A more flexible way to control the mutation speed is through the expression level of Cas9 expression is governed by Doxycycline inducible promoter ${ }^{42}$. As a result, the concentration of editing activity. In Carlin, researchers have shown that the percentage of mutated barcodes

200 increases in samples with high Doxycycline induction, which indicates an increase of CRISPR 201 activity (Fig. S5).

204 beginning of the experiment, the consumption of Dox will lead to a drop of Cas9 concentration 205 over time ${ }^{43}$, leading to a drop in the CRISPR mutation activity. Also, a large proportion of the 206 barcode sequence is deleted over time, which preventing the barcode from further editing (Fig. 207 S5). Therefore, the aggregated result is that the mutation speed decreases exponentially through 
208 time (Fig. 3B, 3C). Contrarily, a different induction method is to add the Dox several times to

209 replenish the concentration of Cas9 protein, which makes the mutation speed steady (Fig. 3B, C).

\section{Leaky Cas9 expression introduces background mutations in the inducible CRISPR}

212 system. The inducible CRISPR system allows users to initiate the lineage recorder at a desirable

213 time point, which enables lineage tracking of a specific developmental process such as cancer

214 metastasis ${ }^{5,6}$. However, one notable drawback is the leakiness of the inducible CRISPR system,

215 in which Cas9 express before the adding Dox ${ }^{42,43}$. The system leakiness can result in an average

216 of $30 \%-50 \%$ of sequences acquire de novo mutations before the Dox induction. Nevertheless, the

217 system still contains abundant unmutated sequences until it saturates at $90 \%$ mutated sequences

218 (Fig. 3D).

219 Moreover, the barcode sequence could be re-targeted by CRISPR-Cas9, resulting in

220 multiple mutations can appear in one sequence. In hgRNA and Carlin, we found the average

221 deletion length increases after Dox induction, suggesting those barcodes have undergone more

222 than one round of mutation (Fig. 3E, Fig. S6). Besides, the initially mutated sequences have

223 shown limited changes on the PAM sequence (Fig. 3F) and a small percentage of deletions (Fig.

224 S6), which further demonstrated that the sequences could be re-targeted by CRISPR. To sum up,

225 although the leaky Cas9 expression brings background noise to the inducible lineage recorder,

226 the system can still accumulate plenty of mutations subsequently. Therefore, the system's

227 leakiness will not significantly sacrifice the lineage recorder's robustness when adjusted

228 correctly. 
230 The lineage-independent mutation causes parallel evolution of barcodes. In genome-editing-

231 based lineage recorders, parallel evolution is almost inevitable when independent lineage

232 acquires the exact same mutation. This causes cells from independent lineage cannot be

233 distinguishable during tree reconstruction (Fig. 4A). In the CRISPR lineage recorder, parallel

234 evolution is amplified by the highly imbalanced mutation distribution from NHEJ. Besides, the

235 recording capacity of the current lineage recorder usually cannot capture the entire cell

236 population detected by the state-of-art single-cell sequencing, which increases the level of

237 parallel evolution. Therefore, an important goal in lineage tracing sequencing analysis is to

238 uncover the distribution of mutation patterns, thereby improving our understanding of parallel

239 evolutions in these lineage recorders.

241 The CRISPR-induced mutations are highly imbalanced. Across all the lineage recorders, we

242 found the most abundant mutation pattern could contribute to up to $60 \%$ of the entire mutation

243 library on average, demonstrating a substantial level of parallel evolution of DNA

244 barcodes. Across all the lineage recorders, the CRISPR-induced indels are overwhelmingly

245 concentrated around the PAM sequence (Fig. 4D, Fig S7-S9). This is most likely due to the

246 highly specified DSB location. For GESTALT and the Carlin, the barcode sequences are target

247 array, which means the deletion of both platforms can be classified into inter-target and intra-

248 target deletions (Fig. 4B). The inter-target deletion occurs when two independent Cas9-guide

249 RNA complexes create two DSB simultaneously, which causes drop-out of all the targets in-

250 between (Fig. 4B). In Carlin, the inter-target deletion and intra-target deletion have a very similar

251 mutation hotspot (Fig. 4E, Fig S7). 
253 Increased mutations diversity improves barcode randomness. In general, increase the

254 diversity of CRISPR mutations can increase the randomness of barcodes ${ }^{44}$, which reduces the

255 number of parallel evolutions. In GESTALT and Carlin, the diversity of barcodes is multiplied

256 by the number of targets in the array. Also, the inter-target deletions further produce additional

257 level of diversity by the number of combinations (Fig. 4C). Similarly, the hgRNA-invivo system

258 uses 60 independent targets throughout the genome to increase the barcode diversity. However,

259 the combinatory effect of multiple barcodes is only detectable via single-cell sequencing.

260 Meanwhile, the hgRNA-invivo system has extended scaffold sequence that allows additional

261 mutations to extend to that region, which generates higher diversity than the canonical guide

262 RNA system (Fig. S9).

263 Across all the lineage recorders, the three mutation types: deletion, insertion, and

264 substitution have significant differences in mutation distribution. We discovered that insertion

265 and inter-target deletion are more random than substitution and intra-target deletion (Fig. 5A, 5B,

266 Fig. S11A), likely due to a second layer diversity generator.

In insertion, the additional diversity is produced by the randomly inserted sequence. In

268 theory, the inserted sequence of length $\mathrm{n}$ has a possibility of $4^{\mathrm{n}}$ possible combination, which

269 generates an exponential increase of diversity. As expected, we discovered that the randomness

270 of insertion patterns increases as the length increase (Fig. S10). In GESTALT and Carlin, the

271 increased diversity of inter-target deletion is generated by the random target-to-target interaction.

272 As a result, the inter-target deletion is more random than intra-target deletion (Fig. 5A, 5B).

273

274 Deletion saturates barcode diversity through time. Among all the mutation types, deletion

275 brings the most irreversible damage to the DNA sequence. When evaluating the accumulation of 
276 each mutation type over time, we discovered that inter-target deletion had gained the most

277 diversity whereas the diversity of insertions and substitutions are gained little or decreased (Fig.

278 5C, Fig. S11). This is mainly because the DNA barcode is gradually saturated by deletion over

279 time as the prolonged CRISPR activity removes a great proportion of the barcode sequence (Fig.

280 S6). As a result, the barcode is too exhausted to bear substitutions and insertions in later

281 development stage. In addition, in GESTALT and Carlin, the inter-target deletion removes all

282 targets in between, which completely clean the diversity of a large proportion of barcode.

283 Contrarily, substitution and insertion have limited diversity when the barcode. In contrast to

284 Carlin, the hgRNA is a single-target platform in which the deletions are shorter. Therefore, the

285 domination of deletion in the mutation library is mitigated compared to GESTALT and Carlin

286 (Fig. S12).

287

288 Discussion

The two properties of CRISPR-induced mutations: speed and rate, have a broad impact

290 on lineage reconstruction. In the non-inducible CRISPR system, the mutation speed depends

291 entirely on the binding between the Cas9 and the target sequence. But in the inducible CRISPR

292 system, the mutation speed can also be controlled by Dox concentration, bringing flexibility and

293 accessibility to the recorder. Moreover, the pulsed induction of Dox enables a stable expression

294 of Cas9, which results in a steady CRISPR mutation speed. The current application of CRISPR

295 lineage recorder usually focuses on a specific developmental process in which the cell division

296 rate is steady. Therefore, pulsed induction of Dox could be useful in many experiments' settings.

297 However, in many organisms, the mutation rate of cell division varies among different tissues

298 and different developmental stages, e.g. mouse embryogenesis ${ }^{45}$. Also, studies have shown that 
299 CRISPR activity varies in different tissues ${ }^{46}$. This could bring challenges to adjusting speed in

300 lineage recorders. Moreover, the current pulsed induction experiment in Carin and hgRNA is

301 only performed in in-vitro systems. In in-vivo experiment, closely monitoring the Cas9

302 expression level could be challenging. Determining the optimal Dox concentration prior to the

303 in-vivo experiment to can help identifying the desired mutation speed.

304 The randomness of CRISPR mutations affects the level of parallel evolutions in lineage

305 recorders. In general, increase the diversity of barcodes by incorporating multiple targets could

306 greatly increase the barcode randomness. We demonstrated that the inter-target interaction of the

307 GESTALT and Carlin platform generated increased the mutation diversity, thus provide robust

308 lineage barcoding through time. The hgRNA mouse model used 60 independent targets

309 throughout the genome to increase the barcode diversity. However, the current experiment

310 readout by bulk DNA sequencing cannot detect the independent target interactions, which could

311 be evaluating using single-cell sequencing.

313 saturate the DNA sequence too quickly, preventing insertion and substitution from occurring.

314 Moreover, inter-target deletion could dropout existing mutations on the sequence, thereby reduce

315 the barcode diversity. Therefore, when building the lineage tree in GESTALT and Carlin, it is

316 important to modeling the dropout effect of mutations.

318 lineage recorders. Recently, many variants of CRISPR-based genome editing technologies have

319 been developed. For example, the base editor ${ }^{49-52}$ brings a single nucleotide change to the

320 genome that could provide sustainability to the barcode. 
One limitation of our mutation distribution analysis is the lack of quantitative measures.

322 Although several studies have demonstrated that NHEJ-induced mutation patterns can be

323 predicted in-silico ${ }^{24,35-38}$, these machine learning models cannot be directly applied to the

324 CRISPR lineage tracing dataset. First, the NHEJ mechanism varies among different organisms,

325 which results in different mutation patterns ${ }^{36}$. Second, these models are trained on a single-target

326 canonical guide RNA library. It cannot capture the properties of target array or hgRNA system.

327 Nevertheless, from the lineage tracing dataset, we discovered the characteristics of CRISPR-

328 induced mutations are mostly concordant with the previously identified characteristics, such as

329 the prevalence of 1-bp insertion. More importantly, we found the mutation frequencies are highly

330 correlated between replicate, which means a machine learning solution could be applied to

331 predict the mutation distribution.

333 phylogenetic algorithms ${ }^{50,51}$. Also, the CRISPR-induced mutations require a novel computational

334 modeling framework. Unsurprisingly, every new lineage reconstruction algorithm has realized

335 the importance of mutation rate. However, directly estimating the mutation rate from the lineage

336 tracing data is one of the biggest challenges, which causes them to creatively detoured the

337 problem. For example, the GAMPL algorithm uses Markov chain to model mutation generation.

338 Instead of estimating the mutation rate, GAMPL used lumped matrix to estimate the transition

339 rate between meta-states. In contrast, Cassiopeia obtained the mutation rate through experiment.

340 They performed an in-vitro experiment of CRISPR barcoding for 15 cell cycles. Then mutation

341 rates are derived empirically using frequency. Although TraceQC does not directly provide an

342 estimation of mutation rate, we believe it could be obtained from a combination of in-vitro

343 experiment and in-silico mutation frequency prediction. Next, an integration between the 
344 sequencing analysis pipeline and tree reconstruction algorithms could produce a more accurate

345 lineage reconstruction strategy.

Overall, the single-cell resolution CRISPR lineage tracing is a promising technology with

347 many potential applications. We developed the TraceQC package to provided sequencing

348 analysis and quality evaluation of lineage tracing datasets across multiple platforms. We hope

349 our study could facilitate a wider application of this technology and provide some insights into

350 its future development.

\section{Methods}

353 TraceQC pipeline The TraceQC R package is available at https://github.com/LiuzLab/TraceQC.

354 The tutorial of TraceQC is available at https://github.com/LiuzLab/TraceQC/wiki. The input of

355 TraceQC pipeline are raw sequencing files and annotated barcode construct sequence. The

356 workflow is shown in Fig. S13. The output is CRISPR-induced mutations and various plots of

357 quality evaluation.

359 Sequencing alignment. The first step of TraceQC is to align reads to the reference sequence.

360 The reference sequence must be annotated with a CRISPR targetable region because the raw

361 reads typically contain adapter sequence. The Needleman-Wunch sequencing alignment with

362 affine gap penalty is applied to each raw sequence. This alignment requires the user to provide

363 four parameters: match, mismatch, gap open penalty, and gap extension penalty. The default

364 parameters are: match $=2$, mismatch $=-2$, gap open $=-6$, gap extension $=-0.1$. After the

365 sequencing alignment, the CRISPR targetable region is selected for the next step. 
Alignment quality evaluation. The alignment quality filter is used to remove reads that are contaminated. In every CRISPR lineage recorder, the barcode sequence is usually amplified and sequenced specifically. However, some samples could contain up to $20-40 \%$ reads that do not come from the CRISPR barcode. Using the alignment score difference, it is usually easy to

371 separate the contaminated sequence from the mutated CRISPR barcode (Fig. S14C). To

372 quantitively determine the contaminated sequence, a decoy sequence library is generated by

373 randomly substitute $\varepsilon$ percentage of the sequence. Next, TraceQC trains a local regression model

374 between the substituted percentage and the alignment score. Finally, the sequence below the 375 optimal substitute percentage can be selected.

377 Identify CRISPR-induced mutations. After the sequencing alignment, the CRISPR targetable

378 region of each sequence is extracted. Next, TraceQC scans through the targetable region of each 379 sequence and extracts every mutation. Basically, TraceQC extracts four properties for each mutation: mutation type (insertion, deletion, and substitution), starting position, indel length,

381 altered sequence. Finally, the TraceQC summarizes every mutation into a count table.

383 Read merging. In single-cell RNA sequencing, a unique molecular identifier (UMI) is used to 384 distinguish unique mRNA transcripts. The cell barcode is used to determine cell identity. 385 Presumably, when the CRISPR barcode is transcribed, the mRNA transcript from each cell 386 should be identical. Therefore, TraceQC provides merging functions for mutations in each cell. 387 First, for each cell, reads with the same UMI are grouped together. The mutations that appears in 388 more than $50 \%$ of reads are retained. During this step, UMI with a low read count should be 389 filtered according to the guideline of the particular single-cell sequencing platform. Next, for 
each UMI in each cell, a second merger is applied to retain mutations that appear in more than $50 \%$ UMI.

QC plots. There are three main aspects that TraceQC evaluates: 1) The quality of sequencing

394 alignment. Using alignment score, TraceQC removes the percentage of contaminated sequences.

395 The method is described in the section: alignment quality evaluation. 2) Mutation patterns can be

396 evaluated from the circular plot. Users can visualize the position of each mutation and identify

397 the mutation hotspots. 3) For single-cell lineage recorders. TraceQC determines the read count

398 distribution of UMI, which allows users to filter. Also, the mutation distribution across cells

399 allows users to understand the lineage recording ability.

400

401 Processing of bulk DNA-seq datasets. In this study, we analyzed bulk DNA-seq datasets from

402 Carlin, GESTALT, hgRNA-invitro, and hgRNA-invivo platforms. The bulk DNA-seq dataset of

403 Carlin used paired-end sequencing. We merged read R1 and R2 using software FLASH with

404 default parameters. Next, the dataset is processed as follows:

405 1. The merged read is processed using the TraceQC alignment with the default parameter.

406 We used $\varepsilon=0.4$ to filter out contaminated sequences.

407 2. We normalized each sequencing sample using count per million (CPM). Reads with $\mathrm{CPM}>10$ are retained.

3. CRISPR-induced mutations are identified using TraceQC. 
413 cover approximately $60 \%$ of the entire barcode, whereas Carlin covers $100 \%$. Therefore, we

414 processed the R1 and R2 of GESTALT separately using the same procedure. Finally, the

415 mutation event of R1 and R2 are merged for each sample.

416 The hgRNA-invitro dataset used single-end sequencing. The complete dataset is

417 processed using the same procedure as Carlin.

418 The hgRNA-invivo platform contains 60 independent CRISPR barcodes. A DNA

419 identifier is assigned to each barcode which presumably cannot be edited by CRISPR. Therefore,

420 we first used the 12 base pairs DNA sequence upstream to locate the DNA identifier. In this step,

421 the Levenshtein distance of 1 is allowed for the 12-bp sequence. Next, the 10-bp directly

422 downstream is extracted to match with the identifier. The sequences are grouped by each

423 identifier and processed by the TraceQC pipeline using the same procedure as Carlin.

425 Processing of single-cell $R \boldsymbol{N}$ A-seq datasets. In this study, we analyzed single-cell RNA-seq

426 datasets from Carlin, LINNAEUS, and scGESTALT. The single-cell dataset of Carlin used 10X

427 Chromium sequencing. First, the raw sequences of CRISPR barcodes are processed with cell

428 ranger V3.1, in which the error-corrected cell barcodes and UMI are identified. Next, the dataset

429 is processed as follows:

430 1. The merged read is processed using the TraceQC alignment with the default parameter.

431 We used $\varepsilon=0.4$ to filter out contaminated sequences.

432 2. For each cell, reads are grouped by UMI. UMIs with read count $<10$ are filtered out.

433 3. CRISPR-induced mutations are identified for each read using TraceQC. 
4. To merge reads in each cell. First, for each UMI, mutations appear in more than $50 \%$ retained. CRISPR barcodes are processed with cell ranger V2.0.2, in which the error-corrected cell barcodes and UMI are identified. However, the read length of 10X single-cell RNA-seq cannot cover the entire barcode region. Therefore, we performed semi-global sequencing alignment that does not penalize the end gap. The other processing pipeline is the same as single-cell Carlin. sequence of GESTALT. According to the annotation, we first used the 10 base pairs DNA sequence downstream to extract the UMI. The rest procedure is the same as single-cell Carlin.

Mutation properties analysis. Using the TraceQC identified mutations, we first calculated the number of unique mutations per sample. Each unique mutation is characterized by mutation type

451 (insertion, deletion or substitution), starting index of the mutation according to the reference

452 sequence, mutation length and altered sequence (only for insertion and substitution). The ternary 453 plot shows the relative ratio of unique mutations in each type.

455 Time-series data analysis. Across all the datasets we analyzed, only Carlin and hgRNA-invitro 456 have time-series data. Briefly, the Carlin platform used CRISPR to target the embryonic stem 
457 cells (ESCs) of mouse. In the one-time induction experiment, the ESCs were exposed with low

$458(0.04 \mu \mathrm{g} / \mathrm{ml})$, medium $(0.20 \mu \mathrm{g} / \mathrm{ml})$ and high $(1.0 \mu \mathrm{g} / \mathrm{ml})$ of Dox. Then, bulk DNA sequencing is

459 performed at 0 hours (before Dox induction), 12-hours, 24-hours, 48-hours, 72-hours and 96-

460 hours. In the pulsed-induction experiment of Carlin, the ESCs is exposed to three pulses of Dox

$461(0.04 \mu \mathrm{g} / \mathrm{ml})$ every 6 hours. After each exposure, cells were picked for sequencing and further

462 outgrowth.

463 The experiment design of hgRNA-invitro is similar. In the one-time induction

464 experiment, the 293T human cell line was exposed to Dox $(2.0 \mu \mathrm{g} / \mathrm{ml})$. Then, bulk DNA

465 sequencing is performed at 0 days (before Dox induction), 2 days, and 14 days. In the pulsed-

466 induction experiment of hgRNA, the 293T cells were first exposed to two hours of Dox to create

467 the founder clone. Next, the Dox is removed while the founder clone grew into 6 clones. To

468 further expand the cell populations, 100 cells are selected for further expansion by exposing

469 them to Dox for two hours. In our study, we compared the mutation speed of one-time induction

470 and pulsed induction of A21 barcode (Fig. 3B, 3C).

471 As for mutation speed analysis, we calculated the percentage of the unmutated sequence

472 out of each sample. Next, we locate the PAM (NGG) sequence of both platforms. Next, we

473 considered the PAM is mutated when either of the two guanines is mutated. When calculating

474 the percentage of PAM for Carlin, all 10 PAMs are treated equally.

475

476 Mutation hotspot analysis. For GESTALT and Carlin target array, the construct sequence

477 designs are similar in which each target (contains spacer and PAM) is separated by a short linker

478 sequence. For both platforms, we defined each target as each consecutive sequence that contains

479 spacer, PAM, and linker. To classify deletion into intra-target deletion and inter-target deletion, 
480 we simply determined if the starting position and ending position belong to the same target. As

481 for the mutation position analysis, the heatmap and histogram show the average result of all

482 samples.

483

484 Mutation dependency analysis. In the mutation dependency analysis, first, we selected an

485 independent experiment of Carlin and hgRNA-invitro before induction because the CRISPR

486 mutations in these samples are least confounded by cell development. Next, for Carlin, scCarlin,

487 GESTALT, scGESTALT, we calculated the mutation dependency between samples that were

488 not taken from the same animal/cell populations.

490 Data and code availability

491 All raw sequencing data in this study is publicly available and can be downloaded from GEO.

492 The corresponding GEO accession number is listed in Table 1. The TraceQC R package is

493 available in GitHub (https://github.com/LiuzLab/TraceQC) under the MIT license. The code for

494 regenerate the analysis results of this manuscript is available at

495 https://github.com/LiuzLab/TraceQC-manuscript.

496

497 Competing interests

498 The authors declare no competing interests.

\section{References}

501 1. Kester, L., \& van Oudenaarden, A. (2018). Single-cell transcriptomics meets lineage tracing. Cell stem cell, 23(2), 166-179. 
2. McKenna, A., \& Gagnon, J. A. (2019). Recording development with single cell dynamic lineage tracing. Development, 146(12), dev169730.

505

3. Wagner, D. E., \& Klein, A. M. (2020). Lineage tracing meets single-cell omics:

506 opportunities and challenges. Nature Reviews Genetics, 21(7), 410-427.

4. Quinn, J. J., Jones, M. G., Okimoto, R. A., Nanjo, S., Chan, M. M., Yosef, N., ... \& metastasis in cancer xenografts. Science, 371(6532).

5. Zhang, W., Bado, I. L., Hu, J., Wan, Y. W., Wu, L., Wang, H., ... \& Zhang, X. H. F. (2021). The bone microenvironment invigorates metastatic seeds for further dissemination. Cell, 184(9), 2471-2486.

6. Bado, I. L., Zhang, W., Hu, J., Xu, Z., Wang, H., Sarkar, P., ... \& Zhang, X. H. F. (2021). cells. Developmental cell, 56(8), 1100-1117.

7. Simeonov, K. P., Byrns, C. N., Clark, M. L., Norgard, R. J., Martin, B., Stanger, B. Z., ... \& Lengner, C. J. (2021). Single-cell lineage tracing of metastatic cancer reveals selection of hybrid EMT states. Cancer Cell.

8. McKenna, A., Findlay, G. M., Gagnon, J. A., Horwitz, M. S., Schier, A. F., \& Shendure, J. (2016). Whole-organism lineage tracing by combinatorial and cumulative genome editing. Science, 353(6298). 
10. Spanjaard, B., Hu, B., Mitic, N., Olivares-Chauvet, P., Janjuha, S., Ninov, N., \& Junker, J. P. (2018). Simultaneous lineage tracing and cell-type identification using CRISPRCas9-induced genetic scars. Nature biotechnology, 36(5), 469-473. (2018). Whole-organism clone tracing using single-cell sequencing. Nature, 556(7699), 108-112. A., ... \& Camargo, F. D. (2020). An engineered CRISPR-Cas9 mouse line for simultaneous readout of lineage histories and gene expression profiles in single cells. Cell, 181(6), 1410-1422.

13. Chan, M. M., Smith, Z. D., Grosswendt, S., Kretzmer, H., Norman, T. M., Adamson, B., ... \& Weissman, J. S. (2019). Molecular recording of mammalian embryogenesis. Nature, 570(7759), 77-82. targeting CRISPR-Cas in human cells. Science, 353(6304). barcodes. Nature methods, 14(2), 195-200. (2018). Developmental barcoding of whole mouse via homing CRISPR. Science, 361(6405). elements. Nature communications, 10(1), 1-9. 
18. Bétermier, M., Bertrand, P., \& Lopez, B. S. (2014). Is non-homologous end-joining really an inherently error-prone process?. PLoS genetics, 10(1), e1004086.

19. Lieber, M. R. (2010). The mechanism of double-strand DNA break repair by the nonhomologous DNA end-joining pathway. Annual review of biochemistry, 79, 181-211. Cas9 for genome engineering. Cell, 157(6), 1262-1278. A programmable dual-RNA-guided DNA endonuclease in adaptive bacterial immunity. Science, 337(6096), 816-821.

22. Hsu, P. D., Scott, D. A., Weinstein, J. A., Ran, F. A., Konermann, S., Agarwala, V., ... \& Zhang, F. (2013). DNA targeting specificity of RNA-guided Cas9 nucleases. Nature biotechnology, 31(9), 827-832.

24. Chen, W., McKenna, A., Schreiber, J., Haeussler, M., Yin, Y., Agarwal, V., ... \& Shendure, J. (2019). Massively parallel profiling and predictive modeling of the outcomes of CRISPR/Cas9-mediated double-strand break repair. Nucleic acids research, 47(15), 7989-8003. 92.

26. Fitch, W. M. (1971). Toward defining the course of evolution: minimum change for a specific tree topology. Systematic Biology, 20(4), 406-416. 
27. Zafar, H., Lin, C., \& Bar-Joseph, Z. (2020). Single-cell lineage tracing by integrating CRISPR-Cas9 mutations with transcriptomic data. Nature communications, 11(1), 1-14. (2021). Estimation of cell lineage trees by maximum-likelihood phylogenetics. The Annals of Applied Statistics, 15(1), 343-362.

29. Jones, M. G., Khodaverdian, A., Quinn, J. J., Chan, M. M., Hussmann, J. A., Wang, using Cassiopeia. Genome biology, 21(1), 1-27. modeling on CRISPR-coded cell lineages: efficient encoding and optimal reconstruction. bioRxiv, 538488 .

31. Gong, W., Granados, A. A., Hu, J., Jones, M. G., Raz, O., Salvador-Martínez, I., ... \& Meyer, P. (2021). Benchmarked approaches for reconstruction of in vitro cell lineages and in silico models of C. elegans and M. musculus developmental trees. Cell Systems.

32. Hwang, G. H., Yu, J., Yang, S., Son, W. J., Lim, K., Kim, H. S., ... \& Bae, S. (2020). CRISPR-sub: Analysis of DNA substitution mutations caused by CRISPR-Cas9 in human cells. Computational and structural biotechnology journal, 18, 1686-1694. cells using the CRISPR-Cas9 system. Science, 343(6166), 80-84.

34. Bassett, A. R., Tibbit, C., Ponting, C. P., \& Liu, J. L. (2013). Highly efficient targeted mutagenesis of Drosophila with the CRISPR/Cas9 system. Cell reports, 4(1), 220-228. 
35. Chakrabarti, A. M., Henser-Brownhill, T., Monserrat, J., Poetsch, A. R., Luscombe, N. M., \& Scaffidi, P. (2019). Target-specific precision of CRISPR-mediated genome editing. Molecular cell, 73(4), 699-713.

36. Shen, M. W., Arbab, M., Hsu, J. Y., Worstell, D., Culbertson, S. J., Krabbe, O., ... \& Sherwood, R. I. (2018). Predictable and precise template-free CRISPR editing of pathogenic variants. Nature, 563(7733), 646-651.

37. Kim, H. K., Min, S., Song, M., Jung, S., Choi, J. W., Kim, Y., ... \& Kim, H. H. (2018). Deep learning improves prediction of CRISPR-Cpf1 guide RNA activity. Nature biotechnology, 36(3), 239-241.

38. Allen, F., Crepaldi, L., Alsinet, C., Strong, A. J., Kleshchevnikov, V., De Angeli, P., ... \& Parts, L. (2019). Predicting the mutations generated by repair of Cas9-induced doublestrand breaks. Nature biotechnology, 37(1), 64-72.

39. Kuscu, C., Arslan, S., Singh, R., Thorpe, J., \& Adli, M. (2014). Genome-wide analysis reveals characteristics of off-target sites bound by the Cas9 endonuclease. Nature biotechnology, 32(7), 677-683.

40. Fu, Y., Foden, J. A., Khayter, C., Maeder, M. L., Reyon, D., Joung, J. K., \& Sander, J. D. (2013). High-frequency off-target mutagenesis induced by CRISPR-Cas nucleases in human cells. Nature biotechnology, 31(9), 822-826. editing. Nature communications, 6(1), 1-6. 
42. González, F., Zhu, Z., Shi, Z. D., Lelli, K., Verma, N., Li, Q. V., \& Huangfu, D. (2014). An iCRISPR platform for rapid, multiplexable, and inducible genome editing in human pluripotent stem cells. Cell stem cell, 15(2), 215-226. for pre-clinical cancer drug discovery. Nature communications, 11(1), 1-16. reconstruct an accurate cell lineage using CRISPR recorders?. Elife, 8, e40292. development. Oncogene, 24(17), 2877-2898.

46. Port, F., Strein, C., Stricker, M., Rauscher, B., Heigwer, F., Zhou, J., ... \& Boutros, M. (2020). A large-scale resource for tissue-specific CRISPR mutagenesis in Drosophila. Elife, 9, e53865. \& Liu, D. R. (2017). Programmable base editing of $A \bullet T$ to $G \bullet C$ in genomic DNA without DNA cleavage. Nature, 551(7681), 464-471. 
635 50. Xi, Z., Liu, L., \& Davis, C. C. (2016). The impact of missing data on species tree

636 estimation. Molecular biology and evolution, 33(3), 838-860.

637 51. Yang, Z., \& Rannala, B. (2012). Molecular phylogenetics: principles and practice. Nature

638 reviews genetics, 13(5), 303-314.

639

640

641

642

643 
A

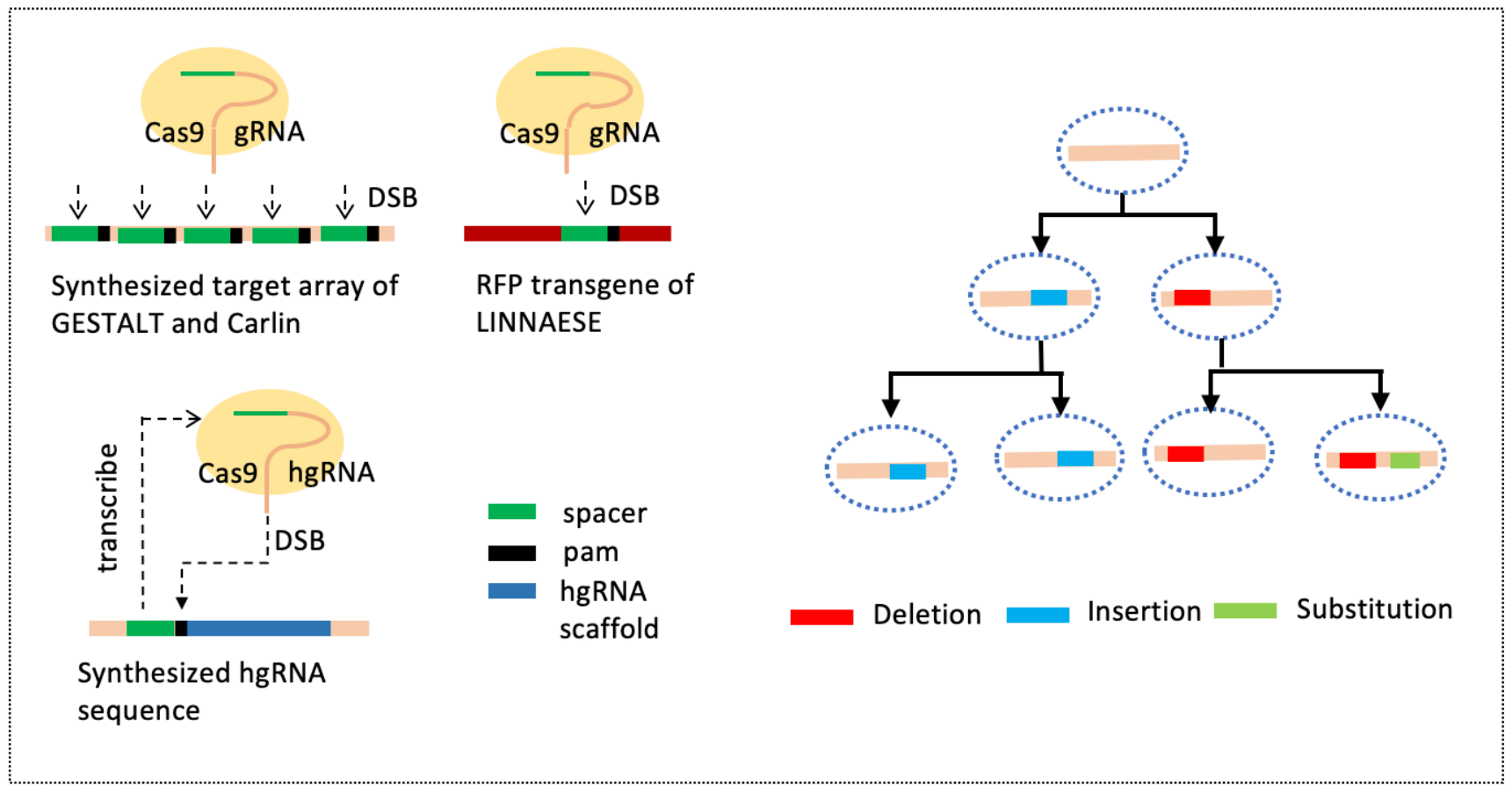

B

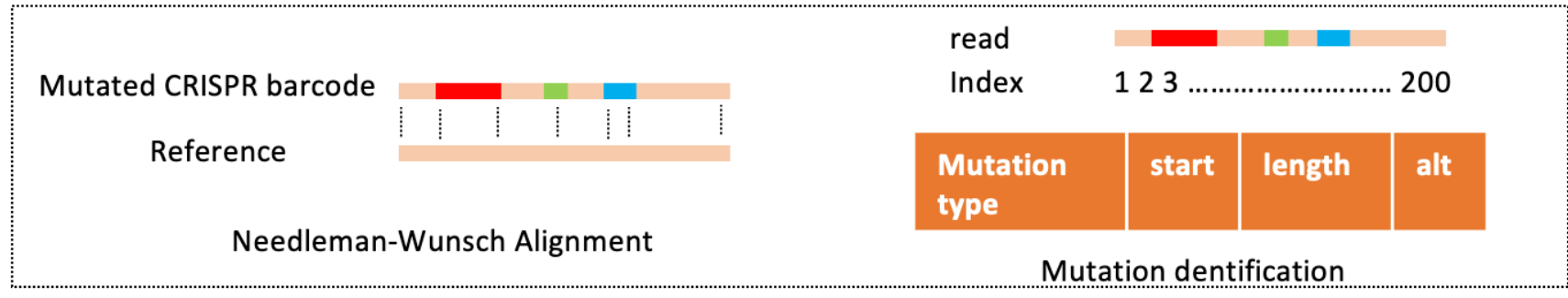

C

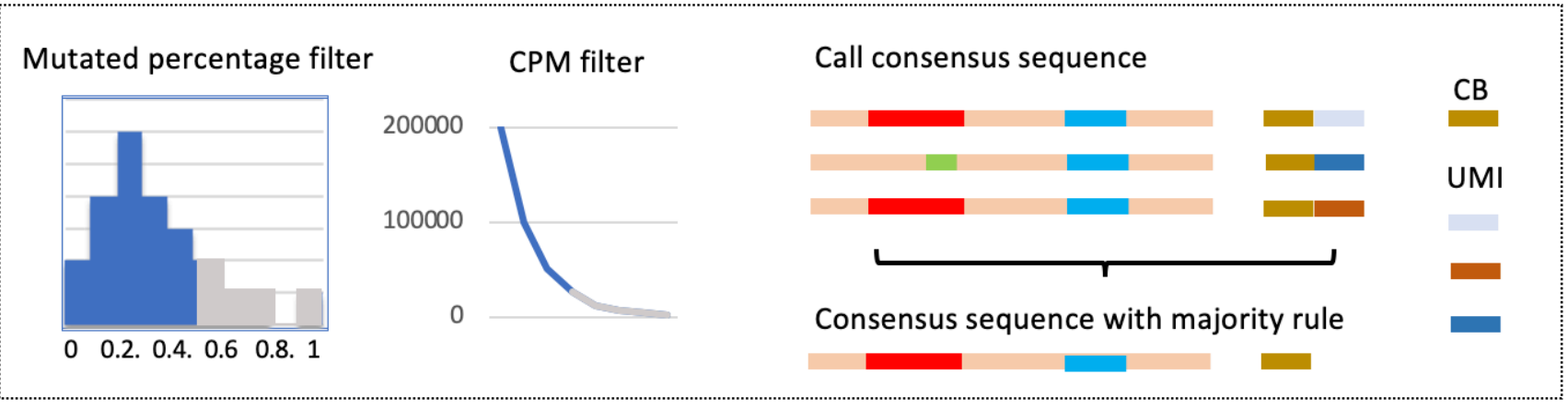

D

Mutation patterns evaluation

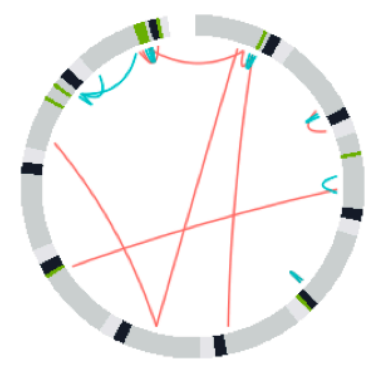

Mutation speed evaluation

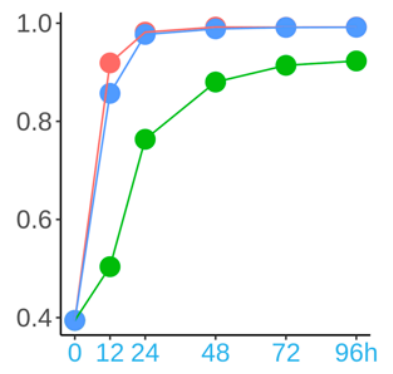

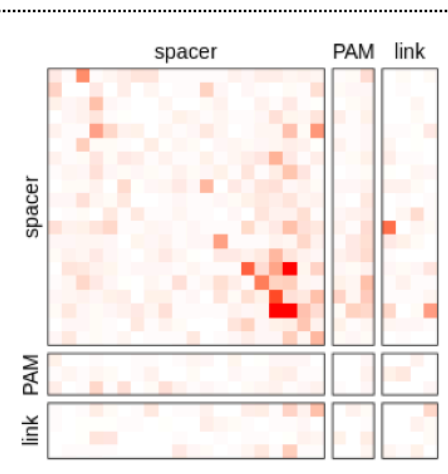

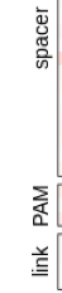


645 Fig. 1 The design of CRISPR lineage recorder and the TraceQC data analysis pipeline. A The 646 three distinctive designs of DNA barcodes. 1) The GESTALT and Carlin platform synthesized

647 multiple targets into one barcode, which is transduced into the model organism's genome. 2) The

648 LINNAESE use CRISPR to edit RFP transgene of an existing zebrafish line zebrabow M. 3) The

649 homing guide system use CRISPR to edit the loci of its own guide RNA. The CRISPR-induced

650 mutations allow tracking of cell development. B The data processing pipeline of TraceQC. First,

651 Needleman-Wunsch sequencing alignment is performed between the reference and the mutated

652 barcode sequence. Second, the mutated read is index according to the reference sequence. The

653 location information is extracted to represent each mutation. $\mathbf{C}$ The denoising procedures of

654 TraceQC. First, the contaminated sequences are filtered based on the sequencing alignment

655 score. Next, the sequences with low abundance are removed to denoise the dataset. Finally, for

656 single-cell data, the sequences are collapsed by UMI and cell barcode. D Various quality

657 assessments of CRISPR-induced mutations using TraceQC package.

\begin{tabular}{|c|c|c|c|c|c|c|c|}
\hline Platforms & Model & Sequencing & Inducible & Target & Datasets & QC results & Ref \\
\hline GESTALT & Zebrafish & DNA-seq & $\mathrm{N}$ & Target array & & Fig. S14-S16 & 8 \\
\hline $\begin{array}{l}\text { hgRNA- } \\
\text { invitro }\end{array}$ & Cell line & DNA-seq & $\mathrm{Y}$ & Single target & & Fig.S17-S18 & 15 \\
\hline $\begin{array}{l}\text { hgRNA- } \\
\text { invivo }\end{array}$ & Mouse & DNA-seq & $\mathrm{N}$ & Single target & & Fig. S19-S20 & 16 \\
\hline scGESTALT & Zebrafish & sc RNA-seq & $\mathrm{N}$ & Target array & & Fig.S21-S22 & 9 \\
\hline LINNEASE & Zebrafish & sc RNA-seq & $\mathrm{N}$ & $\begin{array}{l}\text { RFP transgene } \\
\text { target }\end{array}$ & & Fig. S23-S24 & 10 \\
\hline Carlin & Mouse & $\begin{array}{l}\text { DNA-seq \& } \\
\text { sc RNA-seq }\end{array}$ & $\mathrm{Y}$ & Target array & & Fig. S25-S28 & 12 \\
\hline
\end{tabular}


bioRxiv preprint doi: https://doi.org/10.1101/2021.10.29.466515; this version posted October 30, 2021. The copyright holder for this preprint (which was not certified by peer review) is the author/funder, who has granted bioRxiv a license to display the preprint in perpetuity. It is made available under aCC-BY 4.0 International license.

A

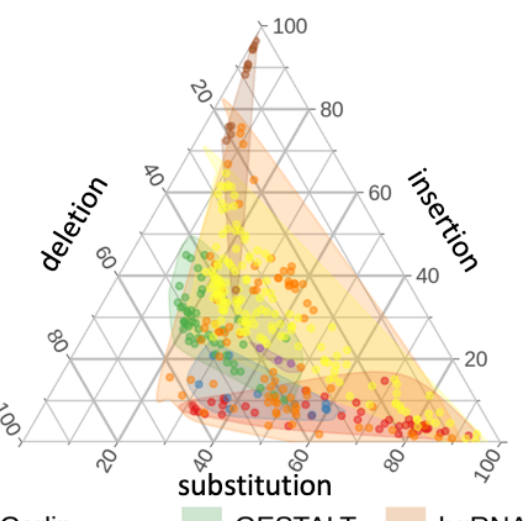

- Carlin - GESTALT - hgRNA-invitro

hgRNA-invivo - LINNAEUS - scCarlin

- scGestalt
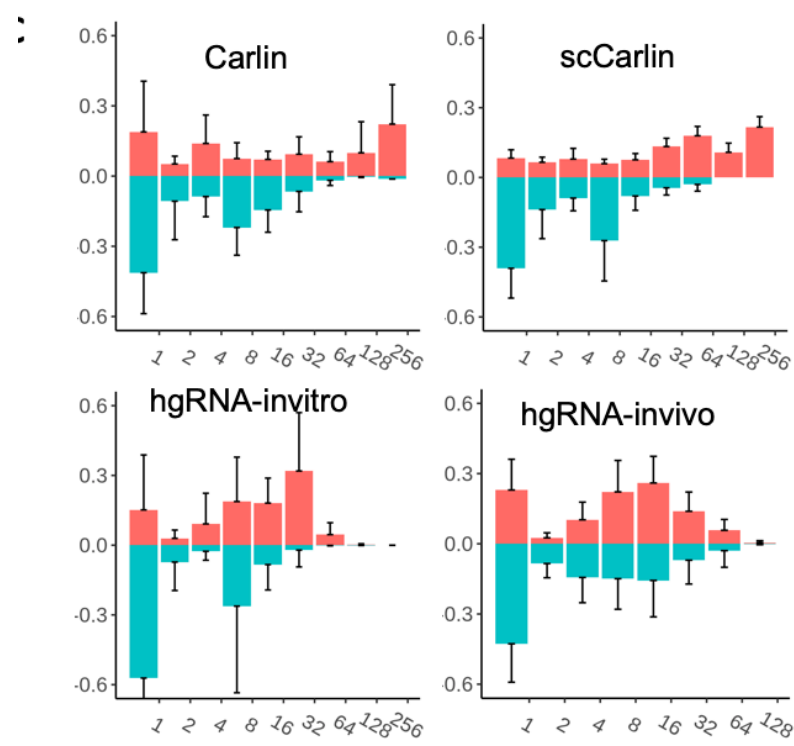

D

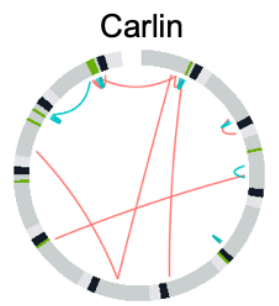

hgRNA-invitro

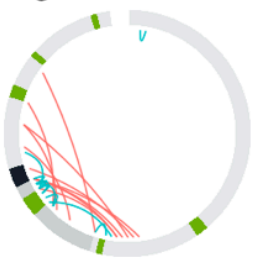

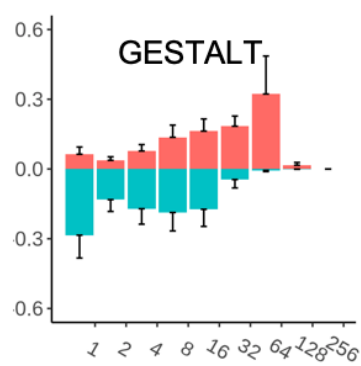

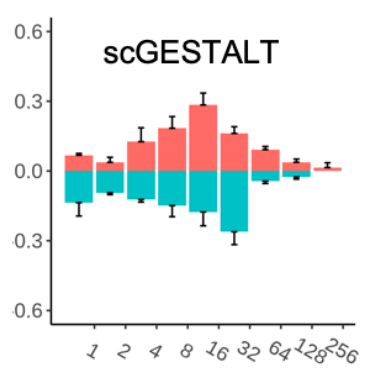

B
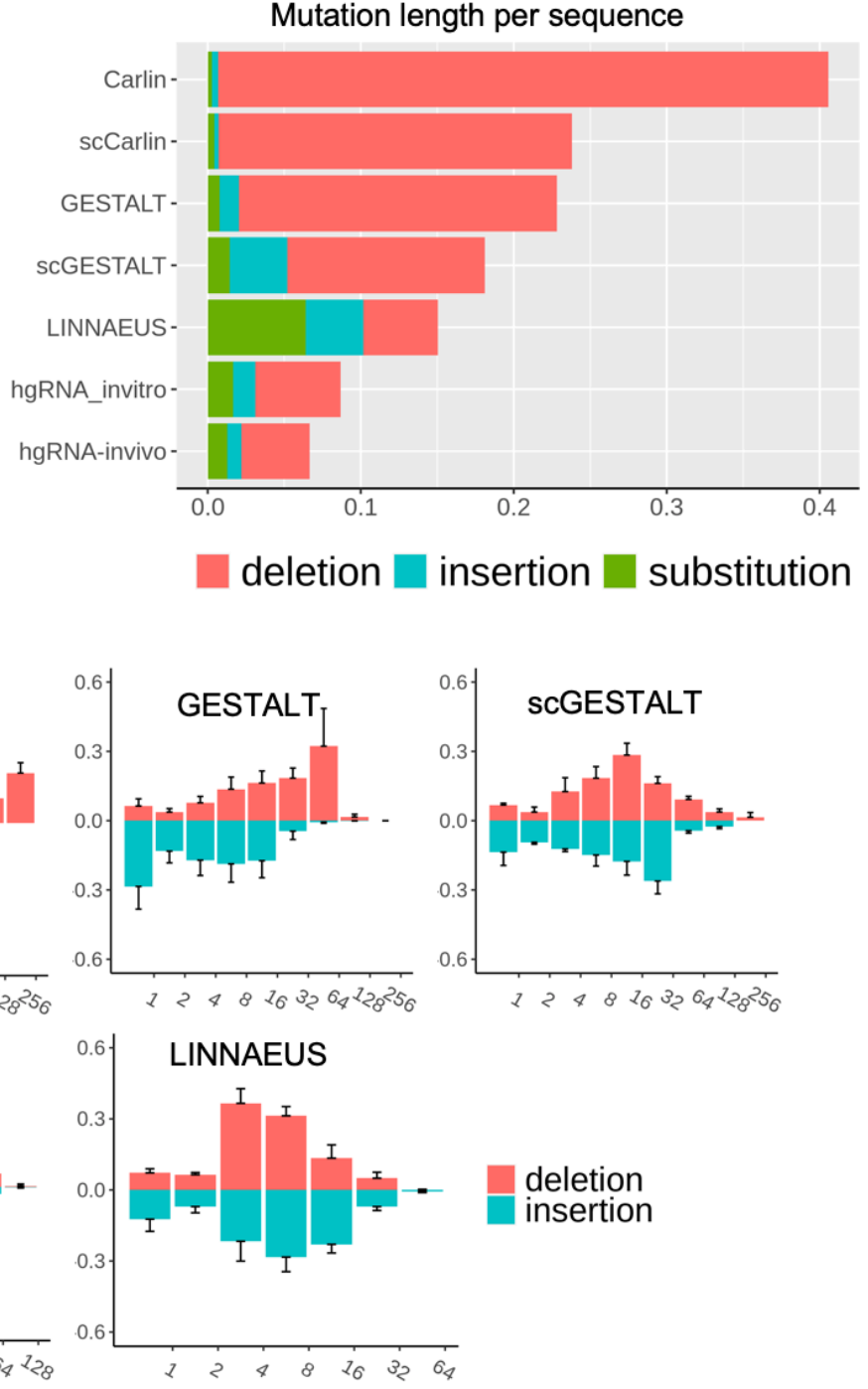

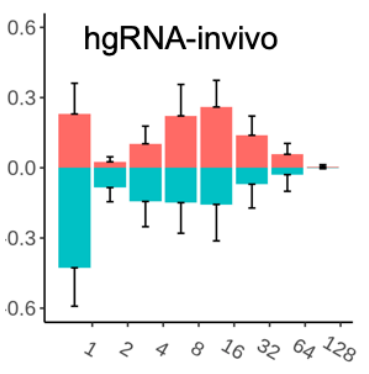

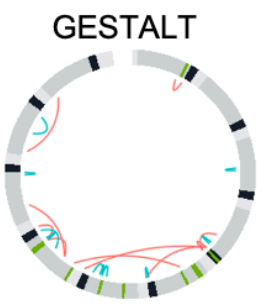

LINNAEUS

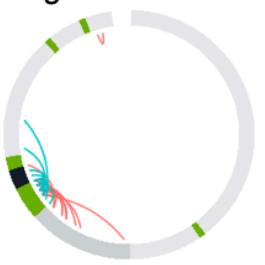

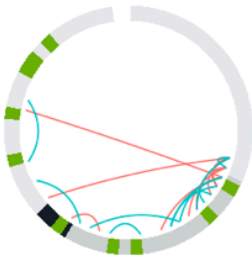

deletion

insertion

substitution

pam

spacer 
664 Fig. 2 Summary of mutations across multiple CRISPR lineage recorders. A The composition

665 percentage of three mutation types: deletion, substitution, and insertion. In the ternary plot, each

666 point is one sequencing sample. B The average percentage of mutated length per sequence. The

667 result is averaged across all the samples in each platform. $\mathbf{C}$ The frequency of indels at a

668 different lengths. The $\mathrm{x}$-axis is the log-scale sequence length by base pair. Each bar aggregate

669 indels length in the range. D The top 10 most abundant insertion, deletion, and substitution

670 patterns of each platform. For each platform, the shown mutations are the intersection result of

671 all samples.

672

673

674

675

676

677

678

679

680

681

682

683

684

685

686 
A
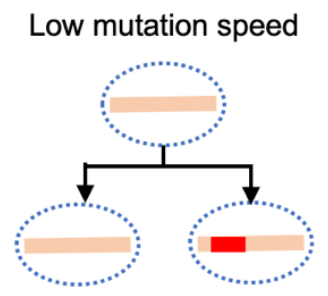

Good mutation speed

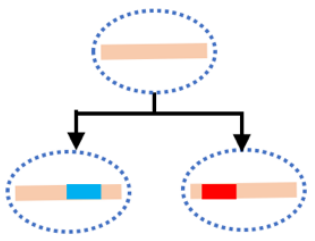

High mutation speed

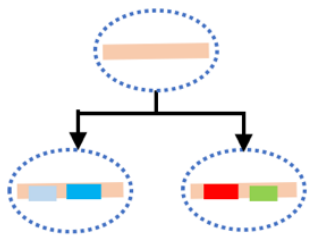

B

Percentage of mutated sequence

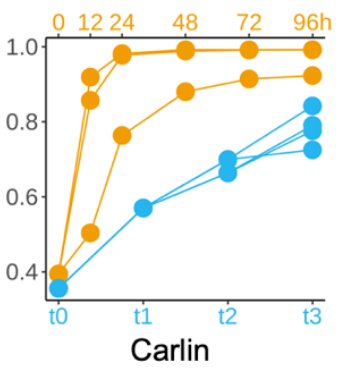

Percentage of mutated sequence

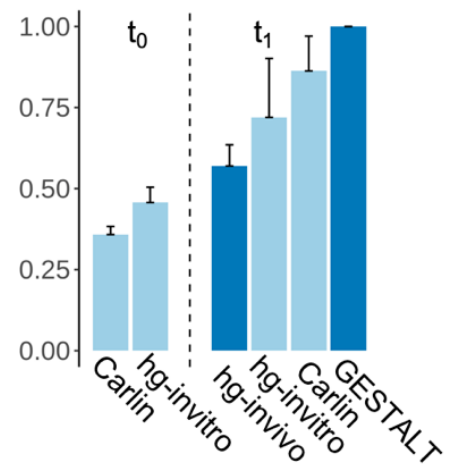

E

Average number of
mutations per sequ

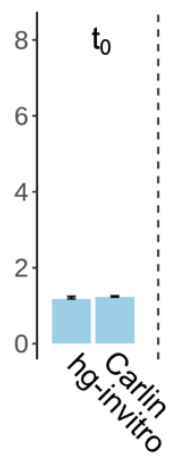

\section{C}

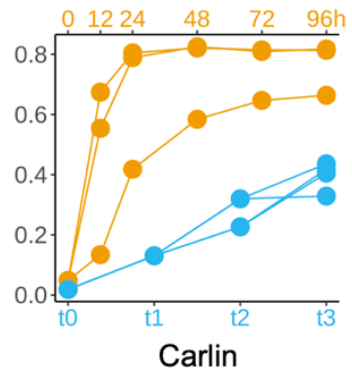

pulsed
Percentage of mutated PAM

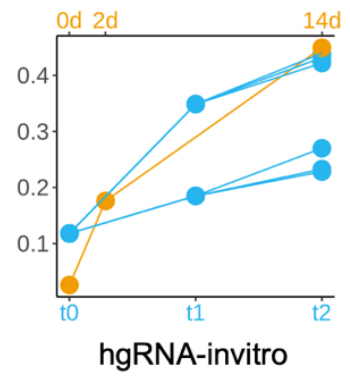

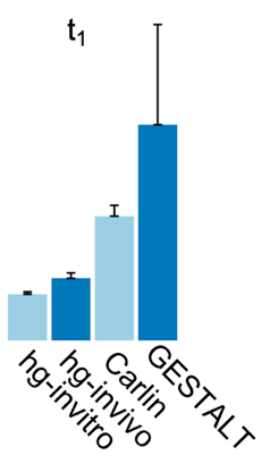

F Percentage of Mutated PAM

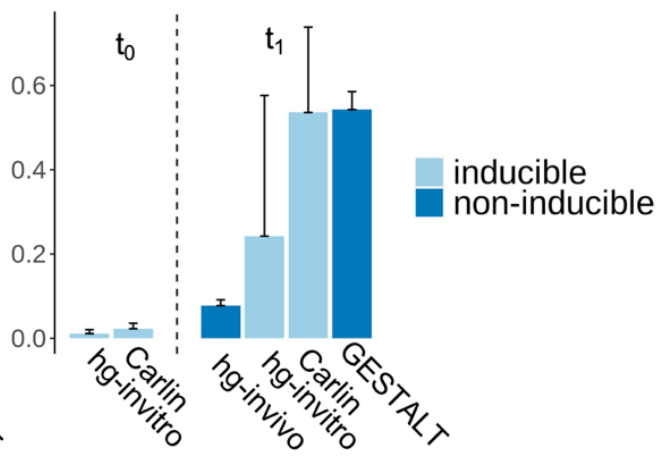

689

690

691

692 
696 Fig. 3 Adjusting mutation speed in CRISPR lineage recorder A An illustration of how mutation

697 speed affect lineage recording. While low mutation speed will not produce enough signals for

698 recording, high mutation speed could saturate the barcode. $\mathbf{B}$ The percentage of the mutated

699 sequence increases through time under two Dox induction methods. The experimental design of

700 Carlin and hgRNA-invitro is described in. In Carlin, the Dox is induced at 0h (one-time), t0, t1,

701 and $\mathrm{t} 2$ (pulsed). In hgRNA-invitro, the Dox is induced at $0 \mathrm{~d}$ (one-time), $\mathrm{t}_{0}$, and $\mathrm{t}_{1}$ (pulsed). The

702 sample at $\mathrm{t} 0$ is sequenced after the Dox induction, which results in a large proportion of mutated

703 barcodes. C The percentage of mutated PAM sequence increases through time. D The percentage

704 of mutated sequence before $\left(\mathrm{t}_{0}\right)$ and after $\left(\mathrm{t}_{1}\right)$ Dox induction. The non-inducible platforms have

705 constitutional Cas9 expression, which is considered equal as after Dox induction. E How many

706 mutations in each sequence before $\left(\mathrm{t}_{0}\right)$ and after $\left(\mathrm{t}_{1}\right)$ Dox induction. F What percentage of PAM

707 sequences are mutated before $\left(\mathrm{t}_{0}\right)$ and after $\left(\mathrm{t}_{1}\right)$ Dox induction.

708

709

710

711

712

713 
bioRxiv preprint doi: https://doi.org/10.1101/2021.10.29.466515. this version posted October 30, 2021. The copyright holder for this preprint (which was not certified by peer review) is the author/funder, who has granted bioRxiv a license to display the preprint in perpetuity. It is made available under aCC-BY 4.0 International license.

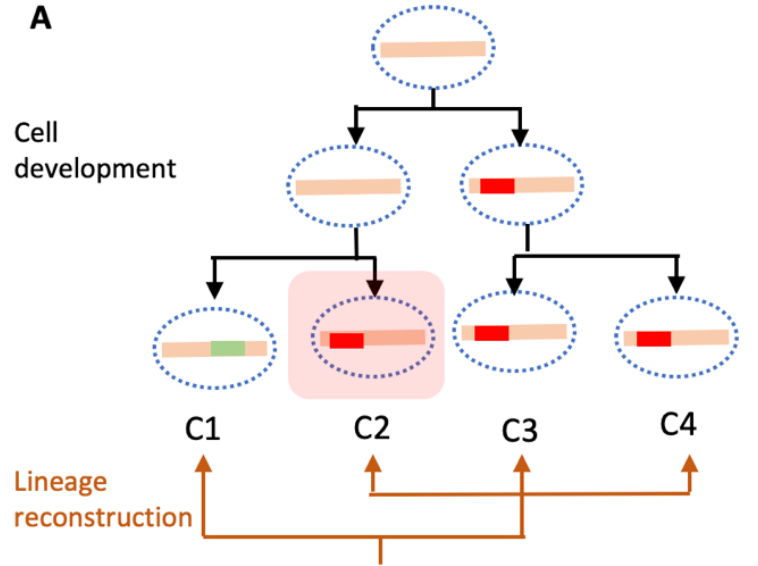

D
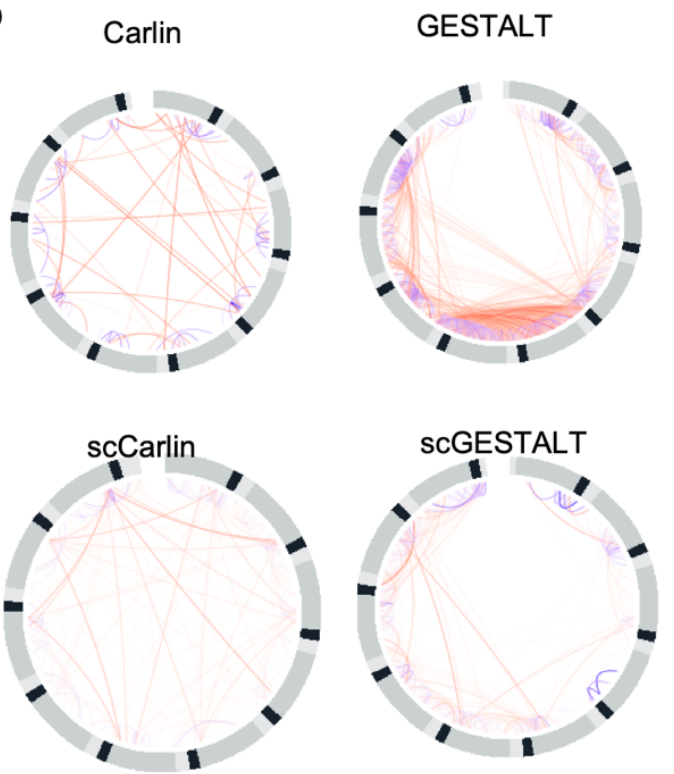

B
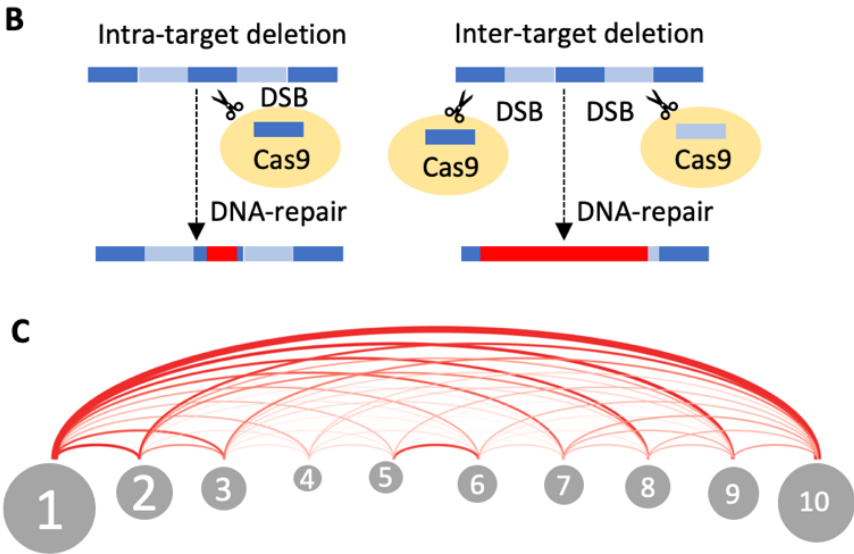

hgRNA-invitro

E

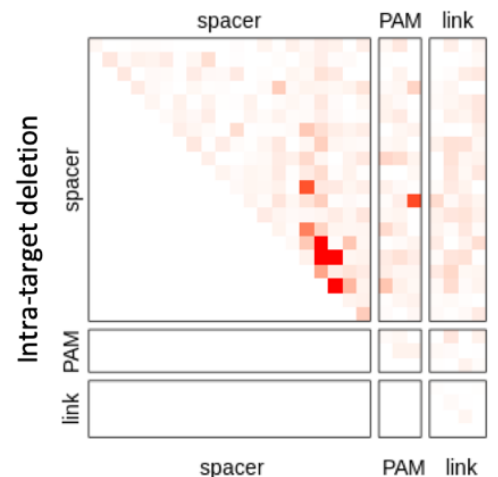

hgRNA-invivo
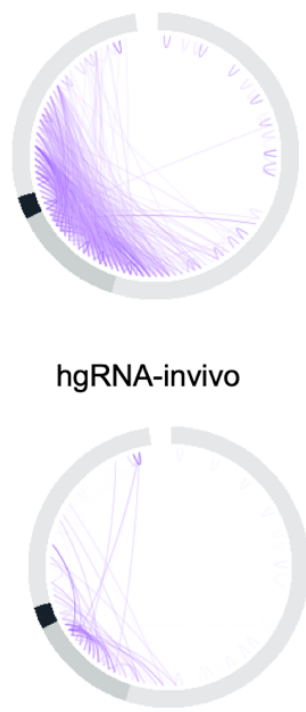

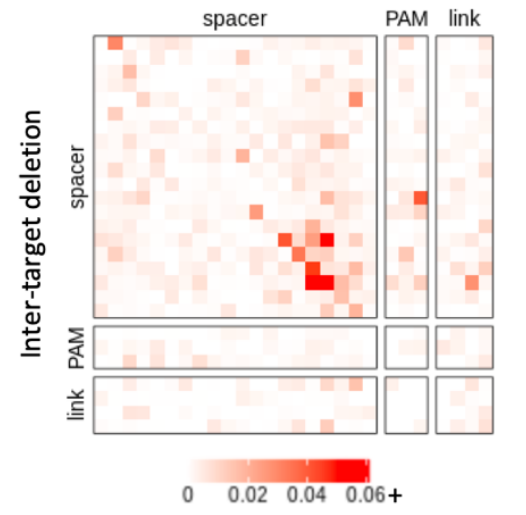

714

715

716

717

718 
720 Fig. 4 Lineage independent mutations cause parallel evolution in CRISPR lineage recorder. A

721 An illustration of parallel evolution leads to undistinguishable lineage assignment. B The

722 mechanism of intra-target and inter-target deletion. $\mathbf{C}$ There are ten targets in the Carlin

723 platform's target array. The arc diagram shows the frequency of target-to-target interaction of

724 inter-target deletions. D The deletion patterns of GESTALT, Carlin, and hgRNA platforms. The

725 red line is inter-target deletion, and the purple line is the intra-target deletion. $\mathbf{E}$ The position of

726 deletion start and deletion end of Carlin.

727

728

729

730

731

732

733

734

735

736

737

738

739

740

741

742 
A

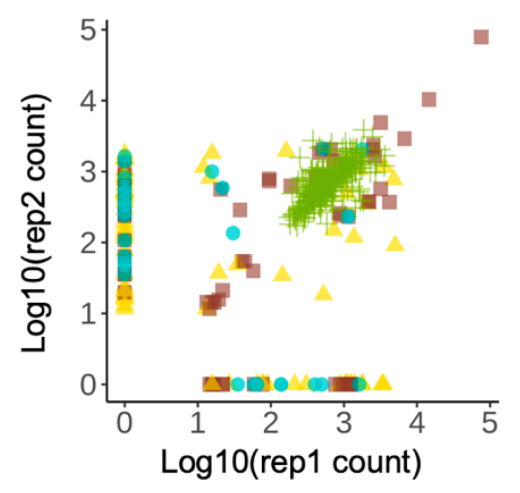

C

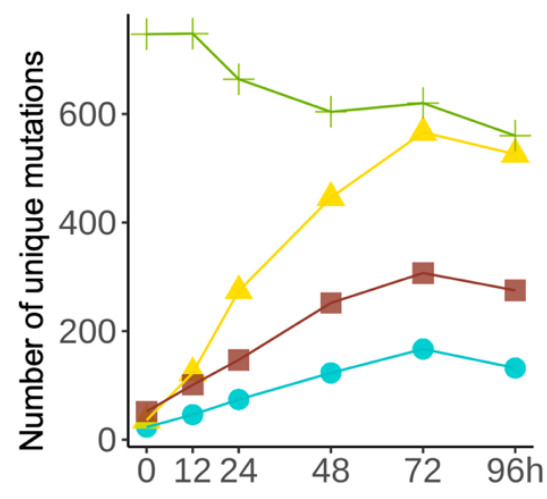

insertion
B
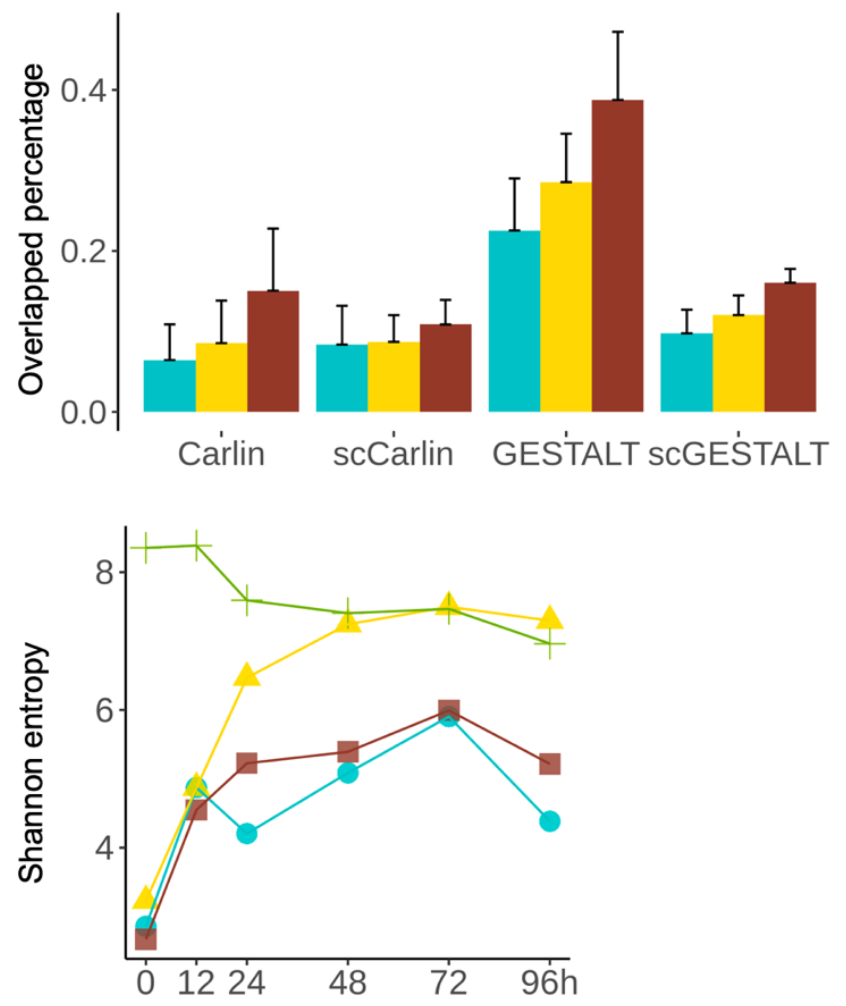

intra-target deletion + substitution

746 Fig 5 The barcode randomness evaluation. A The correlation of replicates of Carlin. The two

747 replicates are negative Dox trial 1 and negative trial 2. The onlapping percentage and Pearson of

748 all mutation types are: inter-target deletion: (19\%, 0.61) intra-target deletion: $(32 \%, 0.82)$

749 insertion: $(14 \%,-0.14)$ substitution $(100 \%, 0.61)$ B The percentage of overlapped sequence

750 mutations between independent samples. The independent experiments are selected from

751 different animals, as they have no lineage relations. C The change of every mutation type over

752 time. The time-series data is Carlin with a low concentration of Dox induction. 